\title{
ANATOMY OF CONSTRUCTION EQUIPMENT MOVE AND ALGORITHMS FOR COLLISION DETECTION
}

\author{
Seokyon Hwang* \\ Department of Civil Engineering, Lamar University, USA \\ *Corresponding author (seokyon.hwang@lamar.edu)
}

\begin{abstract}
Equipment-involved accidents are a long-lasting problem of the construction industry. Collisions between pieces of equipment are responsible for a significant portion of damages resulting from those accidents. This study discusses strategies for preventing such collisions on the construction jobsites. The strategies are built up on two premises: (1) equipment operators can prevent construction equipment from colliding with each other when they are timely aware of approaching equipment in close proximity; and (2) the understanding of the patterns of moves that equipment makes during operation can increase the capability of predicting potential collisions more proactively. Noting that precision tracking technologies can contribute to increasing equipment operator's situation awareness, this study evaluates such technologies in a systematic way so as to identify a technology that is best suited for the proposed strategies. This study analyzes and simplifies the patterns of equipment moves and classifies those into axial-rotation and random-move. The strategies address various collision scenarios for the two patterns, respectively. Incorporating Ultra-wideband (UWB) technology, the strategies allow equipment operators to assess the possibility of collision in real-time.
\end{abstract}

\section{Keywords: Construction Equipment, Equipment Collision, Field Safety, Automated Real-Time Monitoring}

\section{INTRODUCTION}

Safe operation of construction equipment is essential to successful delivery of construction projects, where most, if not all, construction processes involve equipment, and the need for equipment in the processes increases. Despite its indispensable roles in construction, construction equipment frequently presents a considerable amount of risk to projects by causing accidents. Among various types of equipment-involved accidents, the present study is concerned with collisions between pieces of heavy construction equipment on the construction jobsites. It has been reported that accidents involving equipment collision are responsible for a significant portion of fatality in construction (NIOSH 2011); damages resulting from these accidents include injuries, fatalities and monetary and time loss.

Previous studies have identified the lack of timely recognition of dangerous situations as a primary of such accidents (Pratt 2001; Fullerton et al. 2009) - it is not surprising where equipment operators can easily be distracted while operating their equipment in a cluttered work zone. Another significant cause is the increase in nighttime construction - approximately $30 \%$ of highway project in the U.S. is conducted during the night (JolguinVeras et al. 2007); this new trend introduces another challenge to equipment safety where nighttime construction inherently presents equipment operators a problem of limited visibility.

The present research initiative seeks for improvement of jobsite safety by enhancing safety of heavy construction equipment on the jobsites. In line with the goal, the objective of this study is to investigate effective strategies for preventing construction equipment in close proximity form colliding with each other in a more proactive way. In pursuing the research objective, this study conducted the following tasks: (1) identify technologies that can be applied to locate the position of heavy construction equipment in real-time and in precision; (2) analyze and simplify the patterns of moves that construction equipment 
frequently makes during its operation; and (3) investigate strategies for assessing potential collisions proactively.

\section{REVIEW OF POTENTIAL TECHNOLOGIES}

\subsection{Potential Technologies}

Concerning equipment collision due to the lack of situation awareness, an intriguing question within the scope of the present study is: What contributions can the advanced technologies bring to reducing equipment collision on the jobsites? Avoiding such collisions is normally understood to be the responsibility of equipment operators, although it is nearly impossible for them to pay full attention to equipment safety while engaged in construction operation. Under the circumstances, this research notes that some advanced technologies can be effectively applied to increase equipment operators' situation awareness. It is envisioned that operators assisted by enabling technologies can identify dangerous situations more effectively so as to operate equipment more safely. In this context, this study reviewed and compared a few technologies that are expected to increase operators' situation awareness based on a few capacity-related criteria.

\subsection{Comparisons of Technologies}

Real-time tracking is imperative to prevent equipment collisions in a cluttered construction work zone. To date, various technologies have been tested for tracking objects and collecting their spatial data in the field of construction. Based on their capacities with respect to safety, distance, and visibility, some of the technologies have been adopted for automated monitoring of specific targets - to name a few, including a wide range-GPS (Oloufa et al. 2002), LADAR (Teizer et al. 2005), and mobile sensors (Gonzalez-Banos et al. 2002).

Technologies for preventing equipment collision need to satisfy a few technical requirements: it should be able to locate the position of equipment at very short intervals to accommodate equipment at various speeds; it needs to detect the position as accurate as possible in order to assess the possibility of collision reliably; and it should not be influenced by the limitation of line-of-sight in a cluttered work zone. Table 1 lists the technologies that are applied more actively in construction and compares those based on a few key characteristics - line-of-sight, cost, response time, reliability, operation range. The characteristics are critical factors in monitoring and locating the position of equipment on a work zone and to assess the possibility of collision in real-time.

Table 1

Comparison of potential technologies*

Key characteristics: line-of-sight, response Technology time, cost, reliability, and operation range

Infrared Very low cost, small size, fast response;

Not very reliable, very short range

Ultrasonic Low cost, small size, fast response; Not highly reliable, short range

Radar High reliability;

High implementation cost

Vision Wide range;

Low reliability, heavy computation

GPS Unlimited range;

High cost, need for open space

Radio Relatively low cost, no line-of-sight issues; frequency Life of battery, magnetic interruption

*Excerpted and modified from Oloufa et al (2002).

\subsection{Selection of a Technology}

Figure 1 illustrates the process of technology selection that is implemented in the present study. The process evaluates each technology by comparing the capacity of its technology - it uses the characteristics discussed above as selection criteria. Technologies that are inferior to others in terms of the applied criteria are rejected at each step. Concerning the line-of-sight, vision technology is rejected, because line-of-sight can be blocked easily by various objects, even including other equipment, in a cluttered site. Infrared and ultrasonic technologies are rejected at the second step for their short ranges of detection area. Among the three technologies compared at the third step, relatively higher cost of implementation makes GPS technology disqualified. Given the superior capacity in response time and reliability, UWB passed the fourth step to be selected as the best suited technology for preventing collisions between pieces of construction equipment on the construction jobsites. 
Previous studies have recognized a great potential of radio frequency technologies in the field of construction. Most of these studies, however, are limited to locating materials and laborers (Lee et al. 2006; Song et al. 2006; Yagi et al. 2005). The situation is more or less the same for the application of UWB technology. It has not yet been rigorously tested for the purpose of monitoring equipment operations to prevent collisions; rather, it is more applied to simply locate objects of interest. Noting its outstanding capacity, the present study envisions that integrating UWB technology and real-time data analysis can produce a capable method for improving safety of equipment on site.

\section{A SELECTED TECHNOLOGY - UWB}

UWB, a technology for transmitting information spread over a large bandwidth, is often used in precision locating and tracking (FCC 2011). To determine the location of an object, it uses the following techniques (Saeed et al. 2006): the Angle-of-Arrival which measures the angles between a given node and a number of reference nodes; the Received Signal Strength which calculates the distance between two nodes (tags) by measuring the energy of the received signal; and two time-based positioning techniques, Timeof-Arrival and Time-Difference-of-Arrival, which use measurements of travel times of signals between nodes.

While there is a technical similarity between UWB and RFID, UWB technology is known to have a few features superior over RFID: it can transmit more data with less interference than RFID (Mulloy 2004); it has a wider range of coverage, a more accurate measurement of position, and a higher response rate (Jones 2005); and it is more reliable than RFID in metallic and densely packed environments (Jones 2005). These capacities have been brought to the attention of researchers and system developers.

Typical localization systems that are based on UWB technologies normally consist of three components (Venugopal et al. 2010) - a central hub processor, sensors (or receivers), and tags. A tag emits signals at intervals of a certain time. Sensors detect signals from tags and send the detected signals to a central box. Communication between sensors and servers is made via standard network infrastructure, such as Ethernet switches, Wi-Fi access points, and Cat5e shield cables. In order to obtain accurate measurements of spatial data, sensors need to be synchronized. Depending on the number of sensors, the localization system can generate either 2- or 3-dimensional coordinates - to generate 3-dimensional coordinates, a system requires at least four sensors.

\section{ANATOMY OF CONSTRUCTION EQUIPMENT MOVES}

\subsection{Study on equipment collision}

The study of collision has been conducted intensively in the fields of robotics and computer graphics; it is still relatively new in construction. In construction, the focus of collision study falls into three areas of interest: equipment-to-people collision (Pratt et al. 2001; Fullerton et al. 2009), equipment-to-equipment collision (Oloufa et al. 2002), and equipment-to-facility collision (Kim et al 2003; Kang and Miranda 2004).

Regardless of the areas of interest, techniques for collision

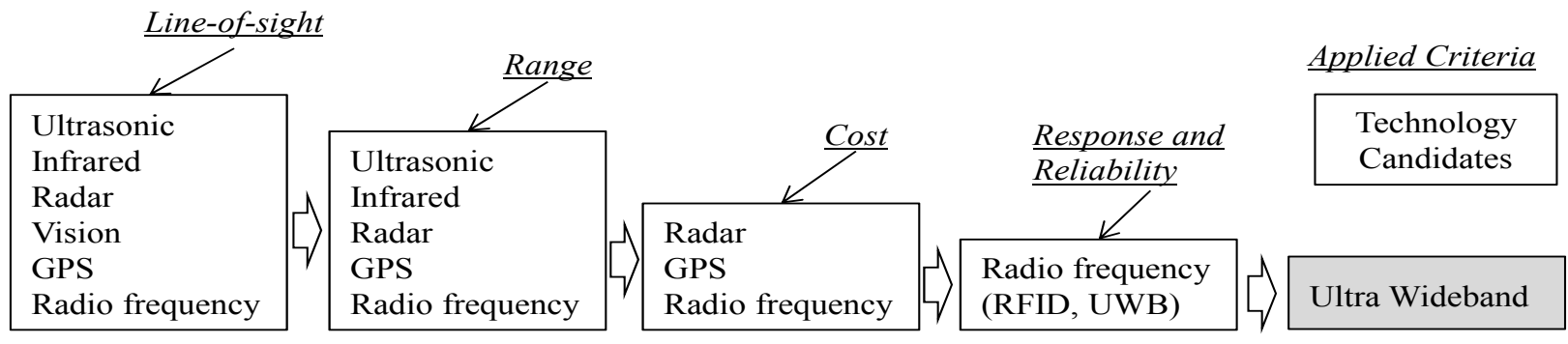

Fig. 1 A process of technology selection

detection need to satisfy the following requirements (Kang and Miranda 2004): sufficient information of geometry and locations of all obstacles, collection of missing data in real-

time by sensors or other devices, and a global model for planning a collision-free path in real-time using sensory data. The first two requirements suggest a need for a 
technology for real-time collection of spatial data. The third highlights the necessity of the assessment of potential collision in advance.

\subsection{Patterns of moves of construction equipment}

There are normally various construction equipment on a jobsite at the same time - some of those are stationary, being placed on a spot and hardly changing its position, while others are moving around the jobsite. The latter are more likely to be involved in collisions. Thus, understanding the moving patterns of equipment is helpful in developing strategies to prevent equipment from colliding with laborers, materials, and other equipment.

While the patterns can be defined in 3-dimensional directions, they can also be simplified in 2-dimensional directions. Construction equipment, other than lifting equipment, move on a 2-dimensional plane - a bulldozer hauls dirt back and forth on a plane. Even lifting equipment frequently makes 2-dimensional moves-a tower crane moves its boom left and right while lifting an object from ground to a position. For this reason, the patterns are simplified in this study into 2-dimensional moves. The simplification makes it possible to reduce the amount of spatial data to be collected and the time needed for computation of data to draw decisions. The benefit of such simplification is significant where both collection and manipulation of data have to be conducted in real-time.

Various moves on a 2-dimensional plane can be further simplified into two patterns as illustrated in Fig. 2. A piece of equipment that is positioned on a spot and rotates around a fixed axis makes "axial-rotation"-tower cranes are a good example. Mobile cranes exhibit a slight difference in a moving pattern where those can move around on a plane. Nevertheless, except that move, a mobile crane also makes "axial-rotation" moves during most of its working time. Another pattern is more commonly observed in a variety of construction equipment, such as trucks and excavators, which can move forward and backward, change their moving directions to left or right, and rotate at one position. In the present study, this

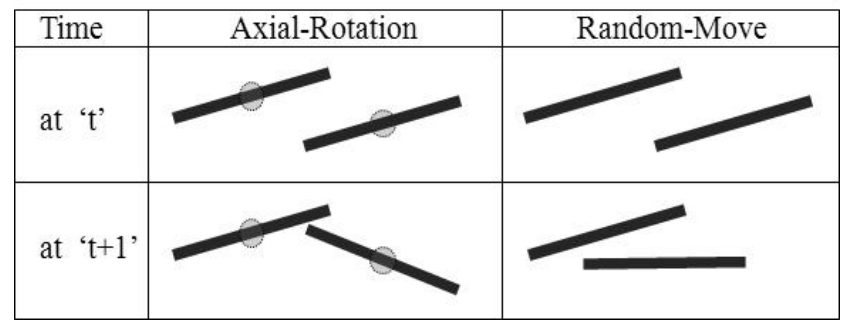

pattern of move is referred to as "random-move." Given the two simplified patterns, a unique strategy for collision prevention is proposed for each pattern.

Fig. 2 Simplified patterns of equipment moves

\section{Strategies for Preventing Collision}

\subsection{Types of collisions}

Types of collisions can be defined as follows: head-to-head, head-to-tail, tail-to-tail, side-to-side, head-to-side, and tailto-side collision. Head of equipment includes any part of the front-end of equipment; tail of equipment includes any part of the rear-end of equipment; and side of equipment can be both left and right. The following details the strategies for tracking equipment using T-type tower crane for "axial-rotation" and trucks for "random-move."

\subsection{A collision-prevention strategy for axial-rotation}

Equipment moving in axial-rotation like a T-type tower crane always follows predetermined moving paths. When the axis of rotation is located at the center of the equipment, it rotates around a circle; if the axis is out of the center, then each end rotates around a different circle. This property allows one to predict moving paths of the equipment over time. As shown in Fig. 3 (a), every possible contact between two booms involves a tip (end) of a boom, resulting in head-to-head, head-to-tail, tail-to-tail, head-to-side, or tail-to-side collision. Thus, it is possible to assess the possibility of collision by locating the ends of booms and measuring distances between a tip of a boom and any part of the other adjacent boom. 


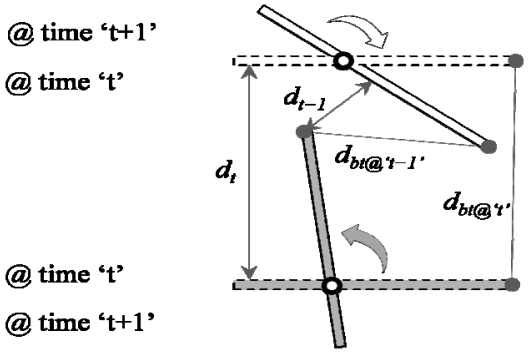

(a) axial-rotation

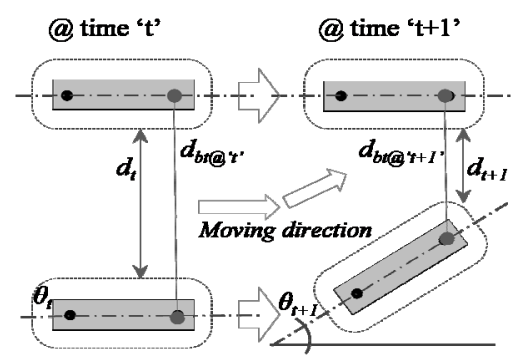

(b) random-move

Fig. 3 Strategies for detecting possible collisions between equipment

Since its moving paths are fixed and known, it is possible to measure the distances, $\left(d_{t}, d_{t+1}\right.$, and so on). Using a CAD drawing of layout plan, the distances can be inferred from the distances between tips of booms. The distances, therefore, can be computed from the positions of UWB tags that indicate the position of the tips of a boom. Given a predetermined distance- $\mathrm{a}$ so-called safety distance, it is possible to identify if two booms are located within a distance that is smaller than or equal to the safety distance by measuring the distance between tags $\left(d_{b t}\right)$.

\subsection{A collision-prevention strategy for random-move}

Fig. 3(b) illustrates a strategy for tracking and assessing possible collisions for random-move using an example of two trucks. This type of equipment normally operates on the ground and has a large mass. It can make a randommove at any time by changing its moving direction-from forward to backward and from left to right; furthermore, it is difficult to predict the moving paths under this condition. There are essentially two ways to attach UWB tags to the body of equipment with a voluminous mass. Four tags may be attached — one at each corner. Alternatively, two tags can be attached along the center-axis of equipmentone at front-end and another at rear-end [Fig. 3(b)]. The latter way can reduce the number of tags needed and the burden of collection and computation of data. Regardless of the number of tags, it is necessary to consider an imaginary boundary area surrounding the equipment in order to deal with its considerable size of mass and unpredictable changes to moving directions. In addition, equipment making random-move can approach to other equipment in close proximity from any directions. Such a high degree of complexity arises from unpredictable moving paths because of possible changes to moving directions at any time. Therefore, tracking the change of directional angle in addition to current location of equipment can allow for predicting potential collisions earlier. The boundary surrounding equipment can also serve as a minimum buffer to absorb a rapid loss of safety distance due to quick turns of equipment in motion.

\section{CONCLUSIONS AND DISCUSSIONS}

A construction jobsite is usually cluttered with various objects. On a site, construction equipment frequently makes various unstructured moves. These conditions often contribute to occurrence of unexpected collisions between equipment in close proximity, which normally results in tremendous damages. Although there could be many approaches to reducing such undesirable incidents, increasing the capability of situation awareness of equipment operators can be a practical and effective way where equipment is ultimately controlled by operators. From this perspective, this study has investigated strategies that can track the positions of equipment over time and assess potential collisions in real-time. The simplified patterns of moves allow simple, yet effective measurement of distances between equipment, which makes it possible to detect potential collisions proactively. The strategies effectively incorporate a radio frequency based precision tracking technology, UWB, to allow real-time collection of spatial data for tracking of equipment positions. 


\section{REFERENCES}

[1] Bohn, J.S., and Teizer, J., "Benefits and barriers of monitoring construction activities using hi-resolution automated camera," Proc., 2009 Construction Research Congress, pp. 21-30, 2009.

[2] Fullerton, C.E., Allread, B.S., and Teizer, J., "Pro-active-real-time personnel warning system," Proc., 2009 Construction Research Congress, pp. 31-40, 2009.

[3] Gonzalez-Banos, H., Lee, C., and Latombe, J. "Real-time combinatorial tracking of a target moving unpredictably among obstacles," Proc., 7th Int. Conference on Robotics and Automation, Vol. 2, pp. 1683-1690, 2002.

[4] Hwang, S., Trupp, T., and Liu, L., "Needs and trends of IT-based construction field data collection," Proc., 4th Joint Int. Symposium on Information Technology in Civil Engineering (CD ROM), 2003.

[5] Jolguin-Veras, J., Ozbay, K., Baker, R., Sackey, D., Medina, A., and Hussain, S., "Toward a comprehensive policy of nighttime construction work," Transportation Research Record, 1861, pp. 117-124, 2007.

[6] Jones, S.K., The Evolution of Modern UWB Technology: A Spectrum Management Perspective, FCC/OET Laboratory, 2005.

[7] Jones, S.K., The evolution of modern UWB technology: A spectrum management perspective, FCC/OET Laboratory, 2005.

[8] Kang, S., and Miranda, E., "Automated Simulation of the Erection Activities in Virtual Construction," Proc. 10th Int. Conference on Computing in Civil and Building Engineering (ICCCBE), pp. 1-12, 2007.

[9] Kim, S., Russell, J., and Koo, K., "Construction robot path-planning for earthwork operations." Journal of Computing in Civil Engineering, Vol. 17(2), pp. 97-104, 2003.

[10] Lee, U., Kang, K., Kim, G., and Cho, H., "Improving tower crane productivity using wireless technology." Computer-Aided Civil and Infrastructure Engr., Vol. 21(8), pp. 594-604, 2006.

[11] Mulloy, R., Ultra wideband RFID technology, Federal Communications Commission RFID workshop, 2004,

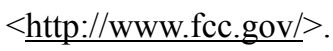

[12] NIOSH, Fatality assessment and control evaluation program, state-based case reports: Highway work zones, National Institute for Occupational Safety and Health, 2011, <http://www2a.cdc.gov/>.

[13] Oloufa A., Ikeda, M. and Hiroshi O., "GPSBased wireless collision detection of construction equipment." Proc., $19^{\text {th }}$ Int. Symposium on Automation and Robotics in Construction, pp. 461-466, 2002.

[14] Pratt, S.G., Fosbroke, D.E., Marsh, S.M., Building safer highway work zones-Measures to prevent worker injuries from vehicles and equipment, National Institute for Occupational Safety and Health, Pub. No. 2001-128, 2001.

[15] Saeed, R.A., Khatun, S., Ali, B.M., and Khazani, M.A., "Performance of Ultra-Wideband Time-of-Arrival Estimation Enhanced With Synchronization Scheme," ECTI Transactions on Electrical Eng, Electronics, and Communications, Vol. 4(1), pp. 78-84, 2006.

[16] Song, J., Haas, C., Caldas, C., Ergen, E., and Akinci, B., "Automating the task of tracking the delivery and receipt of fabricated pipe spools in industrial projects." Automation in Construction, 15 (2), pp. 166-177, 2006.

[17] Teizer, J., Bosche, F., Caldas, C., Hass, C., and Liapi, K., "Real-time, three-dimensional object detection and modeling in construction." Proc., 2005 Int. Symposium on Automation and Robotics in Construction, 2005, < http://www.iaarc.org/>.

[18] Venugoal, M., Cheng, T., and Teizer, J., "Realtime spatial location tracking of construction resources in lay down yards," Proc. 2010 Construction Research Congress, pp. 112-121, 2010.

[19] Yagi, J., Arai, E., and Arai, T., "Parts and packets unification radio frequency identification application for construction." Automation in Construction, Vol. 14 (4), pp. 477-490, 2005. 